\title{
Multishot Codes for Network Coding using Rank-Metric Codes
}

\author{
Roberto W. Nóbrega and Bartolomeu F. Uchôa-Filho \\ Communications Research Group \\ Department of Electrical Engineering \\ Federal University of Santa Catarina \\ Florianópolis, SC, 88040-900, Brazil \\ \{rwnobrega, uchoa\}@eel.ufsc.br
}

\begin{abstract}
The multiplicative-additive finite-field matrix channel arises as an adequate model for linear network coding systems when links are subject to errors and erasures, and both the network topology and the network code are unknown. In a previous work we proposed a general construction of multishot codes for this channel based on the multilevel coding theory. Herein we apply this construction to the rank-metric space, obtaining multishot rank-metric codes which, by lifting, can be converted to codes for the aforementioned channel. We also adapt well-known encoding and decoding algorithms to the considered situation.
\end{abstract}

\section{INTRODUCTION}

Noncoherent linear network coding with unreliable links in a multicast scenario has been given a good deal of attention since the seminal work of Koetter and Kschischang [1]. The problem is suitably modeled by the multiplicative-additive finite-field matrix channel given by

$$
\mathbf{Y}=\mathbf{A X}+\mathbf{Z}
$$

where $\mathbf{X}$ is the $N_{\mathrm{i}}$-by- $T$ channel input matrix, $\mathbf{Y}$ is the $N_{\mathrm{o}^{-}}$ by- $T$ channel output matrix, $\mathbf{A}$ is the $N_{\mathrm{o}}$-by- $N_{\mathrm{i}}$ multiplicative transfer matrix, and $\mathbf{Z}$ is the $N_{\mathrm{o}}$-by- $T$ additive error matrix. All matrices are over some finite field $\mathbb{F}_{q}$.

In the context of network coding, $N_{\mathrm{i}}$ stands for the number of packets transmitted by the source node while $N_{\mathrm{o}}$ stands for the number of packets received by a given sink node at each time slot, a packet consisting of $T$ symbols from $\mathbb{F}_{q}$; the matrices $\mathbf{X}$ and $\mathbf{Y}$ are then formed by juxtaposing the $N_{\mathrm{i}}$ transmitted and the $N_{\mathrm{o}}$ received packets, respectively, seen as row vectors. Matrix A represents the network transfer matrix, which depends on the network topology and network code used (both assumed unknown in a noncoherent scenario), and matrix $\mathbf{Z}$ is related to link error 1 . For simplicity, we set $N_{\mathrm{i}}=$ $N_{\mathrm{o}}=N$ throughout this work.

To achieve a reliable communication over this channel, matrix codes are employed. So far, attention has mostly been given to one-shot matrix codes, that is, codes that use the matrix channel (1) only once. In this case, a code is simply a non-empty subset of matrices. Among the existing

${ }^{1}$ If $N_{\mathrm{e}}$ error packets are injected into the network, and if we dispose these packets to form an $N_{\mathrm{e}}$-by- $T$ matrix $\mathbf{Z}^{\prime}$, then we have that $\mathbf{Z}=\mathbf{D} \mathbf{Z}^{\prime}$, for some $N_{\mathrm{o}}$-by- $N_{\mathrm{e}}$ transfer matrix $\mathbf{D}$. This decomposition, although meaningful in a general context, is unimportant here. constructions for one-shot codes, we highlight that of Silva et al. [2], in which matrix codes are obtained from rank-metric codes. Rank-metric codes, in turn, were already studied before (e.g., by Gabidulin [3] and Roth [4]) in distinct contexts.

In contrast, we herein consider blocks of $n$ consecutive uses of the matrix channel (11). Under this framework, a code is now a non-empty subset of $n$-tuples of matrices; we call it an $n$ shot (or multishot) matrix code. Multishot codes for network coding were studied in [5], where a general construction based on the well-known block coded modulation and multilevel code construction of Imai and Hirakawa [6] has been proposed. It has been shown in [5] that multishot codes can correct more errors than one-shot codes, which motivates this work.

In the present paper, we combine the rank-metric approach of Silva et al. with the multilevel code construction of Imai and Hirakawa to obtain multishot codes for network coding. The problem can also be interpreted within the theory of generalized concatenated codes of Blokh and Zyablov [7], and by doing so it is possible to adapt well-known encoding and decoding procedures to the case at hand (cf. [8, Chapter 15]).

We begin in Section $\Pi$ by providing background on concepts from one-shot noncoherent network coding with errors, including an overview of rank-metric codes applied to network coding. In Section [III] we introduce multishot matrix codes. Section IV presents a brief review of the general multilevel theory to construct block codes over arbitrary metric spaces. The main contribution is Section $\nabla$, where we particularize the multilevel theory to the rank-metric space, derive an encoding procedure based on coset partitioning, and adapt a hard-decision multistage decoding algorithm to our problem. Finally, Section VI concludes the paper.

\section{BACKGROUND}

\section{A. Error Model}

This work follows the approach of Silva, Kschischang, and Koetter [2], in which the adversities of the matrix channel (1) come in two flavors: the rank-deficiency of the multiplicative transfer matrix and the rank of the additive error matrix. These are constrained to

$$
\operatorname{rankdef} \mathbf{A} \leq \rho \quad \text { and } \quad \operatorname{rank} \mathbf{Z} \leq \tau,
$$


where $\rho$ and $\tau$ are integer parameters. While $\rho$ upper bounds the collective effect of unfortunate choices of linear combination coefficients for the network code, wrong min-cut estimation, and packet erasures, $\tau$ measures the maximum number of error packets injected into the network.

\section{B. Matrix Codes}

To fulfill an error-free communication over the matrix channel defined by (1) even under the adversities described in (2), matrix codes are employed. A (one-shot) matrix code $\mathcal{X}$ is a non-empty subset of $\mathbb{F}_{q}^{N \times T}$. Its code rate is given by

$$
R(\mathcal{X})=\frac{\log _{q}|\mathcal{X}|}{N T}
$$

with $0 \leq R(\mathcal{X}) \leq 1$

Let $\mathbf{X}$ be any codeword of a matrix code $\mathcal{X} \subseteq \mathbb{F}_{q}^{N \times T}$ and $\mathbf{Y}$ be the corresponding channel output according to (1). A code $\mathcal{X}$ is said to be $(\rho, \tau)$-correcting if $\mathbf{X}$ can be unambiguously determined from $\mathbf{Y}$ for all choices of $\mathbf{A}$ and $\mathbf{Z}$ subject to (2).

\section{Subspace Coding}

In [2], Silva et al. obtained a sufficient condition for the success of one-shot matrix codes under the presumed error model. Their result asserts that a one-shot matrix code $\mathcal{X}$ is $(\rho, \tau)$-correcting if $d_{\mathrm{S}}(\langle\mathcal{X}\rangle)>2(2 \tau+\rho)$. In this inequality,

$$
\langle\mathcal{X}\rangle=\{\langle\mathbf{X}\rangle: \mathbf{X} \in \mathcal{X}\}
$$

(where $\langle\mathbf{X}\rangle$ stands for the vector subspace spanned by the rows of matrix $\mathbf{X})$ is the subspace code obtained from $\mathcal{X}$, while $d_{\mathrm{S}}(\langle\mathcal{X}\rangle)$ is the minimum subspace distance of $\langle\mathcal{X}\rangle$. The subspace distance between two subspaces $U$ and $V$ is defined as

$$
d_{\mathrm{S}}(U, V)=\operatorname{dim}(U \dot{+} V)-\operatorname{dim}(U \cap V)
$$

where $U \dot{+} V$ is the sum subspace and $U \cap V$ is the intersection subspace. This result reinforces the idea of transmission via subspace selection (i.e., subspace coding), as proposed in [1].

We note that in [9], Silva and Kschischang obtained a necessary and sufficient condition for a matrix code $\mathcal{X}$ to be $(\rho, \tau)$-correcting, namely, $d_{\mathrm{I}}(\langle\mathcal{X}\rangle)>2 \tau+\rho$, where $d_{\mathrm{I}}(\cdot, \cdot)$ is called the injection distance. Nevertheless, we still stick to the subspace distance for mathematical simplicity. Moreover, the proposed multilevel construction gives rise to a multishot matrix code with constant-dimension spanned subspaces, in which case the injection distance and the subspace distance are essentially the same.

\section{Rank-Metric Approach to Network Coding}

In [2], Silva et al. proposed a method to design one-shot matrix codes based on rank-metric codes [3], [4]. A rankmetric code is a block code $\mathcal{R} \subseteq \mathbb{F}_{q^{M}}^{N}$ in which the metric of concern is the rank distance (as opposed to the Hamming distance). The rank distance between $\mathbf{u}, \mathbf{v} \in \mathbb{F}_{q^{M}}^{N}$ is defined as

$$
d_{\mathrm{R}}(\mathbf{u}, \mathbf{v})=\operatorname{rank}(\underline{\mathbf{v}}-\underline{\mathbf{u}}),
$$

where $\underline{\mathbf{u}} \in \mathbb{F}_{q}^{N \times M}$ is the matrix whose rows are the $M$-tuples representing each of the elements of $\mathbf{u} \in \mathbb{F}_{q^{M}}^{N}$ according to some fixed basis for $\mathbb{F}_{q^{M}}$ over $\mathbb{F}_{q}$; the rank distance is indeed a metric [3]. A matrix code $\mathcal{X} \subseteq \mathbb{F}_{q}^{N \times T}$ can be obtained from a rank-metric code $\mathcal{R} \subseteq \mathbb{F}_{q^{M}}^{N}$ by means of a simple operation called lifting, denoted by $\mathcal{I}(\cdot)$ and defined by 2

$$
\begin{aligned}
\mathcal{I}: \mathbb{F}_{q^{M}}^{N} & \longrightarrow \mathbb{F}_{q}^{N \times T} \\
\mathbf{u} & \longmapsto[\mathbf{I} \mid \underline{\mathbf{u}}],
\end{aligned}
$$

where $\mathbf{I}$ is the $N \times N$ identity matrix and $T=N+M$.

The significance of the lifting operation resides in the fact that, for $\mathbf{u}, \mathbf{v} \in \mathbb{F}_{q^{M}}^{N}$,

$$
d_{\mathrm{S}}(\langle\mathcal{I}(\mathbf{u})\rangle,\langle\mathcal{I}(\mathbf{v})\rangle)=2 d_{\mathrm{R}}(\mathbf{u}, \mathbf{v}) .
$$

Thus, if $\mathcal{R} \subseteq \mathbb{F}_{q^{M}}^{N}$ is a rank-metric code then $\mathcal{X}=\mathcal{I}(\mathcal{R}) \subseteq$ $\mathbb{F}_{q}^{N \times T}$ (obtained by lifting each codeword of $\mathcal{R}$ ) gives rise to a matrix code $\mathcal{X}$ with $|\mathcal{X}|=|\mathcal{R}|$ and $d_{\mathrm{S}}(\langle\mathcal{X}\rangle)=2 d_{\mathrm{R}}(\mathcal{R})$ [2].

The problem of obtaining good one-shot codes for network coding could then be reduced to finding good rankmetric codes. But that latter task was already investigated by Gabidulin [3]. He first proved that the Singleton bound is also valid for rank-metric codes, that is, for every $[N, K, D]$ linear rank-metric code over $\mathbb{F}_{q^{M}}$ the condition $D \leq N-K+1$ must hold; he called codes achieving this bound "maximum rank distance codes." Then he constructed a family of maximum rank distance codes, provided $N \leq M$. Even more, Gabidulin described encoding and decoding algorithms for his constructed codes, mainly based on the Reed-Solomon coding theory [8, Chapter 7].

Again in [2], Silva et al. adapted Gabidulin's decoding algorithm for the matrix channel (1). In other words, a method is proposed to solve the following problem: given a received matrix $\mathbf{Y} \in \mathbb{F}_{q}^{T \times M}$ and a Gabidulin code $\mathcal{R} \subseteq \mathbb{F}_{q^{M}}^{N}$, find $\hat{\mathbf{u}} \in \mathcal{R}$ such that

$$
\hat{\mathbf{u}}=\underset{\mathbf{u} \in \mathcal{R}}{\operatorname{argmin}} d_{\mathrm{S}}(\langle\mathbf{X}\rangle,\langle\mathbf{Y}\rangle)
$$

where $\mathbf{X}=\mathcal{I}(\mathbf{u})$. The first step of the method is to decompose matrix $\mathbf{Y}$ into a triplet $\left(\mathbf{r}, \mathbf{L}^{\prime}, \mathbf{E}^{\prime}\right) \in \mathbb{F}_{q^{M}}^{N} \times \mathbb{F}_{q}^{N \times \mu} \times \mathbb{F}_{q}^{\delta \times M}$, operation therein called reduction (which can be interpreted as the opposite of lifting). Decoding then proceeds similarly to standard Gabidulin decoding of $\mathbf{r}$ for code $\mathcal{R}$, except that side information $\mathbf{L}^{\prime}$ and $\mathbf{E}^{\prime}$ is passed to the decoder. For more details (such as the meanings of matrices $\mathbf{L}^{\prime}$ and $\mathbf{E}^{\prime}$ ) we refer the reader to [2].

\section{Multishot Matrix Codes}

We finally introduce multishot matrix codes for error control in noncoherent network coding.

\footnotetext{
${ }^{2}$ Note that this definition differs from that of Silva et al. [2], where the lifting of a matrix $\mathbf{U}$ is the vector subspace spanned by $[\mathbf{I} \mid \mathbf{U}]$.
} 


\section{A. Motivation}

One of the basic problems in the realm of one-shot matrix coding is to find codes with good rates and good errorcorrecting capabilities. To achieve both goals simultaneously, it may be unavoidable to increase the field size, $q$, or the packet size, $T$. Multishot codes allow for a third possibility: to increase the number of channel uses, $n$.

With that in mind, multishot codes are attractive when the system under consideration is such that it is not possible to change the field and packet size. This is true, for example, in fast-topology changing networks (such as wireless ones), where the transfer matrix doesn't stay the same for much long. Under this circumstance, to obtain codes with better error-correcting capabilities we must spread redundancy across multiple shots. Put another way, when errors occur in a random fashion and $q$ and $T$ are fixed, we must use the matrix channel many times in order to approach the channel capacity.

For a simple example in which a multishot code is capable of detecting more errors when compared with the best one could do by simply repeating one-shot codes, we point the reader to [5].

\section{B. Model and Definitions}

In this work we adopt a block-coding approach, in which the matrix channel (1) is used $n$ times in a row. Our channel model then becomes

$$
\mathbf{Y}_{j}=\mathbf{A}_{j} \mathbf{X}_{j}+\mathbf{Z}_{j}
$$

for $j=0, \ldots, n-1$, with matrices $\mathbf{X}_{j}, \mathbf{Y}_{j}, \mathbf{A}_{j}$, and $\mathbf{Z}_{j}$ retaining their dimensions from the one-shot case. We allow the adversities to be spread in any way among the $n$ time slots:

$$
\sum_{j=0}^{n-1} \operatorname{rankdef} \mathbf{A}_{j} \leq \rho \quad \text { and } \quad \sum_{j=0}^{n-1} \operatorname{rank} \mathbf{Z}_{j} \leq \tau .
$$

An $n$-shot (or multishot) matrix code $\mathcal{X}$ is a non-empty subset of $\left(\mathbb{F}_{q}^{N \times T}\right)^{n}$. Its code rate is defined as the ratio between the amount of information symbols conveyed by the transmission of a codeword and the amount of physical symbols spent by each codeword, that is,

$$
R(\mathcal{X})=\frac{\log _{q}|\mathcal{X}|}{n N T}
$$

we have $0 \leq R(\mathcal{X}) \leq 1$. Akin to the one-shot case, a multishot code $\mathcal{X}$ is said to be $(\rho, \tau)$-correcting if $\left(\mathbf{X}_{0}, \ldots, \mathbf{X}_{n-1}\right)$ can be unambiguously determined from $\left(\mathbf{Y}_{0}, \ldots, \mathbf{Y}_{n-1}\right)$ for all choices of $\left(\mathbf{A}_{0}, \ldots, \mathbf{A}_{n-1}\right)$ and $\left(\mathbf{Z}_{0}, \ldots, \mathbf{Z}_{n-1}\right)$ subject to (6).

\section{The Extended Subspace Distance}

We extend the subspace distance to

$$
d_{\mathrm{S}}(\boldsymbol{U}, \boldsymbol{V})=\sum_{j=0}^{n-1} d_{\mathrm{S}}\left(U_{j}, V_{j}\right),
$$

where $\boldsymbol{U}=\left(U_{0}, \ldots, U_{n-1}\right)$ and $\boldsymbol{V}=\left(V_{0}, \ldots, V_{n-1}\right)$ are $n$ tuples of subspaces of $\mathbb{F}_{q}^{N}$ and $d_{\mathrm{S}}(\cdot, \cdot)$ in the right-hand side is given by (3). We now state a multishot counterpart for the result of Silva et. al presented in Section II.

Theorem 1: Let $\mathcal{X}$ be a multishot matrix code. If $d_{\mathrm{S}}(\langle\mathcal{X}\rangle)>2(2 \rho+\tau)$ then $\mathcal{X}$ is $(\rho, \tau)$-correcting.

Proof: The proof is a simple generalization of [2, Theorem 1]. Let $\boldsymbol{X}=\left(\mathbf{X}_{0}, \ldots, \mathbf{X}_{n-1}\right)$ be the transmitted codeword and $\boldsymbol{Y}=\left(\mathbf{Y}_{0}, \ldots, \mathbf{Y}_{n-1}\right)$ be the received sequence, according to (5). Let $\langle\boldsymbol{X}\rangle=\left(\left\langle\mathbf{X}_{0}\right\rangle, \ldots,\left\langle\mathbf{X}_{n-1}\right\rangle\right)$ and $\langle\boldsymbol{Y}\rangle=$ $\left(\left\langle\mathbf{Y}_{0}\right\rangle, \ldots,\left\langle\mathbf{Y}_{n-1}\right\rangle\right)$. We have

$$
\begin{aligned}
& d_{\mathrm{S}}(\langle\boldsymbol{X}\rangle,\langle\boldsymbol{Y}\rangle)=\sum_{j=0}^{n-1} d_{\mathrm{S}}\left(\left\langle\mathbf{X}_{j}\right\rangle,\left\langle\mathbf{Y}_{j}\right\rangle\right) \\
& \quad \leq \sum_{j=0}^{n-1} d_{\mathrm{S}}\left(\left\langle\mathbf{X}_{j}\right\rangle,\left\langle\mathbf{A}_{j} \mathbf{X}_{j}\right\rangle\right)+\sum_{j=0}^{n-1} d_{\mathrm{S}}\left(\left\langle\mathbf{A}_{j} \mathbf{X}_{j}\right\rangle,\left\langle\mathbf{Y}_{j}\right\rangle\right) \\
& \quad \leq \sum_{j=0}^{n-1} \operatorname{rankdef} \mathbf{A}_{j}+2 \sum_{j=0}^{n-1} \operatorname{rank} \mathbf{Z}_{j} \\
& \quad \leq \rho+2 \tau .
\end{aligned}
$$

Since $\rho+2 \tau<d_{\mathrm{S}}(\langle\boldsymbol{\mathcal { X }}\rangle) / 2$, a minimum extended subspace distance decoder is guaranteed to yield $\langle\boldsymbol{X}\rangle$ given $\langle\boldsymbol{Y}\rangle$.

\section{Multishot Rank-Metric Codes}

Let $\boldsymbol{u}=\left(\mathbf{u}_{0}, \ldots, \mathbf{u}_{n-1}\right)$ and $\boldsymbol{v}=\left(\mathbf{v}_{0}, \ldots, \mathbf{v}_{n-1}\right)$ be two $n$ tuples of vectors in $\mathbb{F}_{q^{M}}^{N}$. The extended rank distance between them is defined by

$$
d_{\mathrm{R}}(\boldsymbol{u}, \boldsymbol{v})=\sum_{j=0}^{n-1} d_{\mathrm{R}}\left(\mathbf{u}_{j}, \mathbf{v}_{j}\right),
$$

where $d_{R}(\cdot, \cdot)$ in the right-hand side is the rank distance as defined in (4). Just like regular rank-metric codes, multishot rank-metric codes can be applied to noncoherent network coding. To this end, we use an extended version of the lifting operation defined as $\mathcal{I}(\boldsymbol{u})=\left(\mathcal{I}\left(\mathbf{u}_{0}\right), \ldots, \mathcal{I}\left(\mathbf{u}_{n-1}\right)\right)$, where $\boldsymbol{u}=\left(\mathbf{u}_{0}, \ldots, \mathbf{u}_{n-1}\right)$. It is straightforward to show that

$$
d_{\mathrm{S}}(\langle\mathcal{I}(\boldsymbol{u})\rangle,\langle\mathcal{I}(\boldsymbol{v})\rangle)=2 d_{\mathrm{R}}(\boldsymbol{u}, \boldsymbol{v}) .
$$

Thus, a multishot rank-metric code $\mathcal{R} \subseteq\left(\mathbb{F}_{q^{M}}^{N}\right)^{n}$ gives rise to a multishot matrix code $\mathcal{X} \subseteq\left(\mathbb{F}_{q}^{N \times T}\right)^{n}$ (where $T=N+M$ ) defined by $\mathcal{X}=\mathcal{I}(\mathcal{R})=\{\mathcal{I}(\boldsymbol{u}): \boldsymbol{u} \in \mathcal{R}\}$, with $|\mathcal{X}|=|\mathcal{R}|$ and $d_{\mathrm{S}}(\langle\mathcal{X}\rangle)=2 d_{\mathrm{R}}(\boldsymbol{\mathcal { R }})$.

\section{General Multilevel Code Construction}

The multilevel code construction was proposed by Imai and Hirakawa [6] in 1977 and became very popular in the 80's and 90 's with more general constructions being developed by many other researchers. Although originally targeted at codes over a given signal set $\mathcal{S}$ of the Euclidean space, the construction can be generalized for block codes over any finite subset $\mathcal{S}$ of a given metric space $\mathcal{M}$ with associated distance $d_{\mathrm{M}}(\cdot, \cdot)$. (This is true as long as the component codes are Hamming-metric.) Next, we base our description of the multilevel construction on the work of Lin and Costello [8, Chapter 19], wherein many references on this subject are listed.

Given a set $\mathcal{S}$, an $m$-level partitioning of $\mathcal{S}$ is defined by a sequence of $m+1$ partitions $\Gamma_{0}, \ldots, \Gamma_{m}$ of $\mathcal{S}$ such that 
$\Gamma_{0}=\{\mathcal{S}\}$ and, for $1 \leq i \leq m$, partition $\Gamma_{i}$ is a refinement of partition $\Gamma_{i-1}$, in the sense that the subsets in $\Gamma_{i}$ are subsubsets of the subsets in $\Gamma_{i-1}$. It is possible to represent an $m$-level partitioning by a rooted tree with $m+1$ levels, labeled from 0 to $m$. The nodes at level $i$ are the subsets in the partition $\Gamma_{i}$. The unique node at level 0 is called the the root node (which is the set $\mathcal{S}$ ) while the nodes at level $m$ are called the leaf nodes. A node $\mathcal{Y} \in \Gamma_{i}$ is a child of the only element $\mathcal{X} \in \Gamma_{i-1}$ such that $\mathcal{Y} \subseteq \mathcal{X}$. Equivalently, a node $\mathcal{Y} \in \Gamma_{i}$ is the parent of every node $\mathcal{Z} \in \Gamma_{i+1}$ such that $\mathcal{Z} \subseteq \mathcal{Y}$.

A level $i$ is said to be nested if every node in this level has the same number $p_{i}$ of children, although we do allow the partitions at level $i+1$ to have different cardinalities. (Note that, by this definition, level 0 is always nested in any partitioning.) In our construction of multishot codes we require level $i$ to be nested for $0 \leq i<m$. The edges joining a subset at level $i$ to subsets at level $i+1$ in the tree can then be labeled with the numbers $0, \ldots, p_{i}-1$. We denote by $Q\left(c^{(0)}, \ldots, c^{(m-1)}\right)$ the subset of nodes in $\Gamma_{m}$ reached by following the path $\left(c^{(0)}, \ldots, c^{(m-1)}\right)$ in the tree, where $0 \leq c^{(i)}<p_{i}$ for $0 \leq i<m$.

Consider now a metric space $\mathcal{M}$ with distance $d_{\mathrm{M}}(\cdot, \cdot)$ and let $\mathcal{S} \subseteq \mathcal{M}$ be a finite subset of $\mathcal{M}$. We now describe the procedure to construct a block code $\mathcal{C}$ over $\mathcal{S}$ of length $n$. Let $\Gamma_{0}, \ldots, \Gamma_{m}$ be an $m$-level partitioning of $\mathcal{S}$, with level $i$ nested for $0 \leq i<m$. We define the intrasubset distance of level $i$ as

$$
d_{\mathrm{M}}^{(i)}=\min \left\{d_{\mathrm{M}}(\mathcal{Y}): \mathcal{Y} \in \Gamma_{i}\right\},
$$

for $0 \leq i<m$. All levels $0 \leq i<m$ must be "protected" by classical codes of length $n$ over $\mathbb{F}_{p_{i}}$, called component codes and denoted by $\mathcal{H}_{i}$, with minimum Hamming distances

$$
d_{\mathrm{H}}^{(i)}=d_{\mathrm{H}}\left(\mathcal{H}_{i}\right) .
$$

The codewords of $\mathcal{C} \subseteq \mathcal{M}^{n}$ are obtained as follows.

1) Form all possible arrays of $m$ rows and $n$ columns

$$
\boldsymbol{\Lambda}=\left[\begin{array}{cccc}
c_{0}^{(0)} & c_{1}^{(0)} & \cdots & c_{n-1}^{(0)} \\
c_{0}^{(1)} & c_{1}^{(1)} & \cdots & c_{n-1}^{(1)} \\
\vdots & \vdots & \ddots & \vdots \\
c_{0}^{(m-1)} & c_{1}^{(m-1)} & \cdots & c_{n-1}^{(m-1)}
\end{array}\right]
$$

where the $i$-th row of $\boldsymbol{\Lambda}$ is a codeword $\mathbf{c}^{(i)}=$ $\left(c_{0}^{(i)}, \ldots, c_{n-1}^{(i)}\right)$ of code $\mathcal{H}_{i}$, for $0 \leq i<m$. The set of all such arrays is denoted by $\mathcal{A}$ and has cardinality $|\mathcal{A}|=\prod_{i=0}^{m-1}\left|\mathcal{H}_{i}\right|$.

2) The $j$-th column $\mathbf{c}_{j}=\left(c_{j}^{(0)}, \ldots, c_{j}^{(m-1)}\right)$ of a given array $\boldsymbol{\Lambda} \in \mathcal{A}$ specifies a path in the rooted tree, starting at $\mathcal{S} \in \Gamma_{0}$ and ending at $Q\left(\mathbf{c}_{j}\right) \in \Gamma_{m}$, for $0 \leq j \leq n$.

3) Each array $\boldsymbol{\Lambda} \in \mathcal{A}$ gives rise to a set of codewords:

$$
\mathcal{C}_{\boldsymbol{\Lambda}}=Q\left(\mathbf{c}_{0}\right) \times Q\left(\mathbf{c}_{1}\right) \times \cdots \times Q\left(\mathbf{c}_{n-1}\right) .
$$

4) Finally, the constructed code $\mathcal{C}$ is the union of all such (disjoint) sets:

$$
\mathcal{C}=\bigcup_{\Lambda \in \mathcal{A}} \mathcal{C}_{\Lambda}
$$

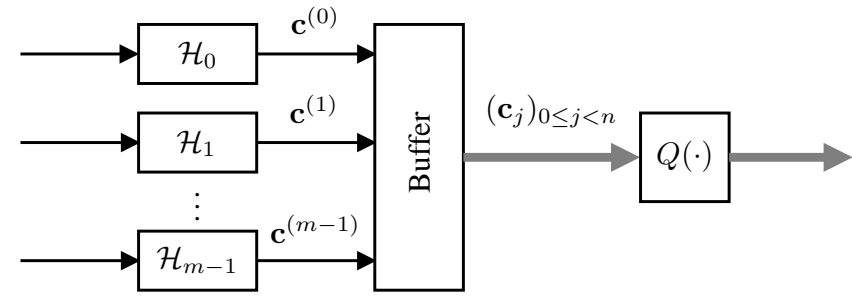

Figure 1. Block diagram for a general multilevel encoder.

Accordingly, the total number of codewords in the constructed code $\mathcal{C}$ is

$$
|\mathcal{C}|=\sum_{\boldsymbol{\Lambda} \in \mathcal{A}} \prod_{0 \leq j<n}\left|Q\left(\mathbf{c}_{j}\right)\right| .
$$

For the case when $|\mathcal{Y}|=1$ for all $\mathcal{Y} \in \Gamma_{m}$, each array $\Lambda \in \mathcal{A}$ gives rise to exactly one codeword and the encoding procedure can be represented by Figure 1. In this case,

$$
|\mathcal{C}|=|\mathcal{A}|=\prod_{i=0}^{m}\left|\mathcal{H}_{i}\right|
$$

Also, from multilevel theory [8], the minimum (extended) distance of the constructed code $\mathcal{C}$ is lower-bounded by

$$
d_{\mathrm{M}}(\mathcal{C}) \geq \min \left\{d_{\mathrm{M}}^{(i)} d_{\mathrm{H}}^{(i)}: 0 \leq i<m\right\} .
$$

\section{Multilevel Construction using RANK-METRIC CODES}

In this section, we aim at constructing a multishot rankmetric code $\mathcal{R} \subseteq\left(\mathbb{F}_{q^{M}}^{N}\right)^{n}$ which, by lifting of each component of its codewords, can be converted to a multishot block matrix code $\mathcal{X} \subseteq\left(\mathbb{F}_{q}^{N \times T}\right)^{n}$ with $T=N+M$ (cf. Section 【II). To this end, we particularize the multilevel construction described earlier to the case where the metric space $\mathcal{M}$ with $d_{\mathrm{M}}(\cdot, \cdot)$ is, in fact, the space $\mathbb{F}_{q^{M}}^{N}$ with the rank distance $d_{\mathrm{R}}(\cdot, \cdot)$. The finite subset $\mathcal{S} \subseteq \mathcal{M}$ will then be a $q^{M}$-ary $[N, K, D]$ linear rank-metric code $\mathcal{R} \subseteq \mathbb{F}_{q^{M}}^{N}$. Furthermore, the multilevel partitioning $\Gamma_{0}, \ldots, \Gamma_{m}$ will be obtained by a technique called coset partitioning [8, Section 4.5], described next.

\section{A. Coset Partitioning}

Let

$$
\mathbf{G}=\left[\begin{array}{cccc}
g_{0,0} & g_{0,1} & \cdots & g_{0, K-1} \\
g_{1,0} & g_{1,1} & \cdots & g_{1, K-1} \\
\vdots & \vdots & \ddots & \vdots \\
g_{N-1,0} & g_{N-1,1} & \cdots & g_{N-1, K-1}
\end{array}\right]
$$

be a generating matrix 3 for $\mathcal{R}$ and let $K=K_{0}>\cdots>K_{m}=$ 0 be a strictly decreasing sequence of integers. For $0 \leq i \leq m$, define $\mathcal{R}_{i}$ to be the linear code generated by $\mathbf{G}\left[0: K_{i}-1\right]$ (i.e., the first $K_{i}$ columns of $\mathbf{G}$ ) and $\overline{\mathcal{R}}_{i}$ to be the linear code generated by $\mathbf{G}\left[K_{i}: K-1\right]$ (i.e., the last $K-K_{i}$ columns of $\mathbf{G})$. Then

$$
\Gamma_{i}=\left\{\mathcal{R}_{i}+\mathbf{v}: \mathbf{v} \in \overline{\mathcal{R}}_{i}\right\}
$$

\footnotetext{
${ }^{3}$ We adopt the convention that the code is the column space of $\mathbf{G}$.
} 


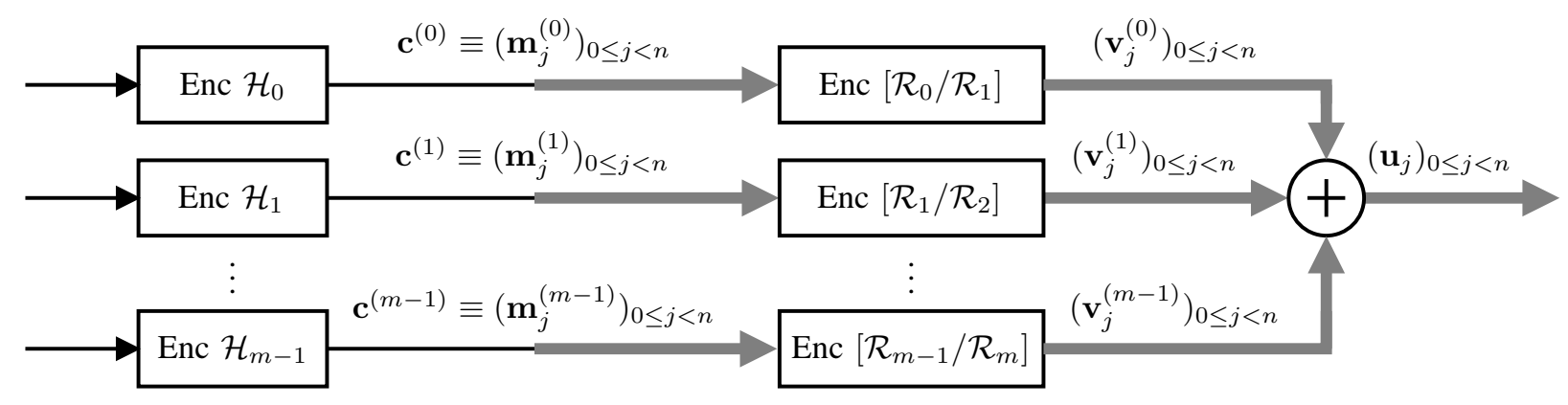

Figure 2. Block diagram for a generalized concatenated coding system.

defines an $m$-level partitioning $\Gamma_{0}, \ldots, \Gamma_{m}$ of $\mathcal{R}$ with minimum intrasubset distances $d_{\mathrm{R}}^{(i)}=d_{\mathrm{R}}\left(\mathcal{R}_{i}\right)$, for $0 \leq i<m$. In other words, $\Gamma_{i}$ consists of cosets of $\mathcal{R}_{i}$ having as coset leaders the elements of $\overline{\mathcal{R}}_{i}$. Note that we have $\Gamma_{0}=\{\mathcal{R}\}$ and $\Gamma_{m}=\{\{\mathbf{u}\}: \mathbf{u} \in \mathcal{R}\}$. Additionally, each level $i$, for $0 \leq i<m$, is nested, with every node at level $i$ having

$$
p_{i}=\frac{\left|\Gamma_{i+1}\right|}{\left|\Gamma_{i}\right|}=\frac{\left(q^{M}\right)^{K-K_{i+1}}}{\left(q^{M}\right)^{K-K_{i}}}=q^{M\left(K_{i}-K_{i+1}\right)}
$$

children.

\section{B. Generalized Concatenation and Encoding Procedure}

The multilevel partitioning $\Gamma_{0}, \ldots, \Gamma_{m}$ just constructed, along with suitable $p_{i}$-ary Hamming-metric component codes $\mathcal{H}_{0}, \ldots, \mathcal{H}_{m-1}$ gives rise to a multishot rank-metric code $\mathcal{R} \subseteq\left(\mathbb{F}_{q^{M}}^{N}\right)^{n}$ with cardinality given by (7) and minimum distance satisfying (8). Nevertheless, the construction-as presented in Section IV_-does not specify any efficient encoding or decoding procedure. To this end, we will make use of the connection between the multilevel coding theory and generalized concatenated codes (also known as multilevel concatenated codes) [8, Chapter 15].

Let $\mathbf{c}_{0}, \ldots, \mathbf{c}_{n-1}$ be the output of the buffer in Figure 1 As said before, each $\mathbf{c}_{j}$ represents a path in the rooted tree starting at $\mathcal{R} \in \Gamma_{0}$ and ending at the leaf $\left\{\mathbf{u}_{j}\right\}=Q\left(\mathbf{c}_{j}\right) \in \Gamma_{m}$. Recall that $p_{i}=q^{M\left(K_{i}-K_{i+1}\right)}$. In view of that, each component $c_{j}^{(i)} \in$ $\mathbb{F}_{p_{i}}$ of $\mathbf{c}_{j}$ can also be viewed as a $\left(K_{i}-K_{i+1}\right)$-tuple with elements in $\mathbb{F}_{q^{M}}$. Denote this tuple by $\mathbf{m}_{j}^{(i)} \in \mathbb{F}_{q^{M}}^{K_{i}-K_{i+1}}$. This suggests us to define

$$
\mathbf{u}_{j} \triangleq \sum_{i=0}^{m-1} \mathbf{G}\left[K_{i+1}: K_{i}-1\right] \cdot \mathbf{m}_{j}^{(i)}=\sum_{i=0}^{m-1} \mathbf{v}_{j}^{(i)}
$$

where each $\mathbf{v}_{j}^{(i)}=\mathbf{G}\left[K_{i+1}: K_{i}-1\right] \cdot \mathbf{m}_{j}^{(i)}$ can be viewed as the codeword associated with message $\mathbf{m}_{j}^{(i)}$ of the linear code generated by matrix $\mathbf{G}\left[K_{i+1}: K_{i}-1\right]$ (i.e., column $K_{i+1}$ up to, and including, column $K_{i}-1$ of $\mathbf{G}$ ). This code is denoted by $\left[\mathcal{R}_{i} / \mathcal{R}_{i+1}\right]$ and, since it contains coset leaders for partition $\mathcal{R}_{i} / \mathcal{R}_{i+1}$ (i.e., $\mathcal{R}_{i+1} \subseteq \mathcal{R}_{i}$ and its cosets), it is called a coset code [8, Section 15.2].

Thus, encoding can be summarized in the following steps, illustrated in Figure 2.

1) Let $\mathbf{c}^{(i)} \in \mathcal{H}_{i}$ be a codeword of $\mathcal{H}_{i}$, for $0 \leq i<m$.
2) Translate each codeword $\mathbf{c}^{(i)}=\left(c_{0}^{(i)}, \ldots, c_{n-1}^{(i)}\right)$ into $\left(\mathbf{m}_{0}^{(i)}, \ldots, \mathbf{m}_{n-1}^{(i)}\right)$.

3) Encode each $\mathbf{m}_{j}^{(i)} \in \mathbb{F}_{q^{M}}^{K_{i}-K_{i+1}}, 0 \leq j<n$, using $\mathbf{G}\left[K_{i+1}: K_{i}-1\right]$ to form $\mathbf{v}_{j}^{(i)} \in \mathbb{F}_{q^{M}}^{N}$.

4) Finally, the $j$-th coordinate of the codeword is calculated according to $\mathbf{u}_{j}=\sum_{i=0}^{m-1} \mathbf{v}_{j}^{(i)}$.

In the terminology of generalized concatenated codes, the component codes $\mathcal{H}_{i}$ are called outer codes, while the coset codes $\left[\mathcal{R}_{i} / \mathcal{R}_{i+1}\right]$ are the inner codes.

\section{A Special Situation}

Consider the special situation in which $m=K$ and $K_{i}=$ $K-i$ in a way that $p_{i}=q^{M}$ for $0 \leq i<m$. If, in addition, (i) every rank-metric code $\mathcal{R}_{i}$ is maximum rank distance, and (ii) every $\left(q^{M}\right.$-ary) component code $\mathcal{H}_{i}$ is maximum Hamming distance separable with distance $d_{\mathrm{H}}^{(i)}=\left\lceil d / d_{\mathrm{R}}^{(i)}\right\rceil$, then we have that

$$
d_{\mathrm{R}}^{(i)}=N-K_{i}+1=N-K+i+1
$$

and

$$
\log _{q^{M}}\left|\mathcal{H}_{i}\right|=n-\left\lceil\frac{d}{d_{\mathrm{R}}^{(i)}}\right\rceil+1 .
$$

The first condition is always achievable with Gabidulin codes whenever $N \leq M$ (this becomes clear from the structure of a generating matrix for Gabidulin codes [3]). The second condition is also achievable if $n<q^{M}$ (e.g., with ReedSolomon codes), which is typically true. Hence, in view of (8) and (7), we get a multishot rank-metric code $\mathcal{R}$ with minimum distance $d_{\mathrm{R}}(\boldsymbol{\mathcal { R }})=d$ and cardinality

$$
\begin{aligned}
\log _{q}|\boldsymbol{\mathcal { R }}| & =\sum_{i=0}^{m-1} \log _{q}\left|\mathcal{H}_{i}\right| \\
& =\sum_{i=0}^{K-1} M\left(n+1-\left\lceil\frac{d}{N-K+i+1}\right\rceil\right) \\
& =M K(n+1)-M \sum_{i=0}^{K-1}\left\lceil\frac{d}{N-K+i+1}\right\rceil,
\end{aligned}
$$

this value-after maximized over all $K \in\{0, \ldots, N\}$-being an upper bound on the size of any multishot rank-metric code constructed using the proposed method. 


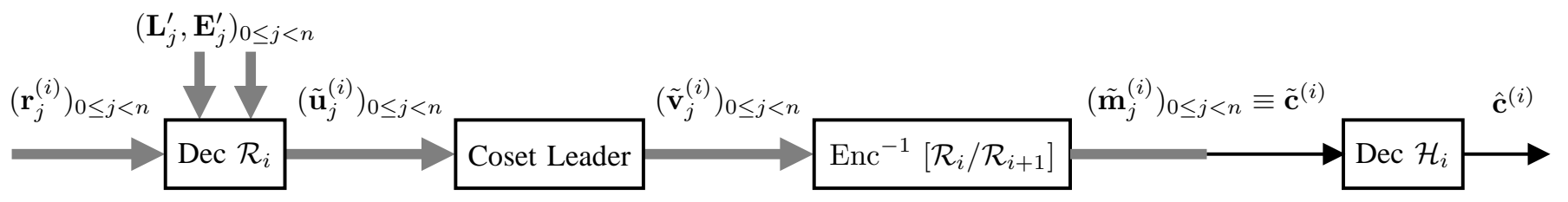

Figure 3. Block diagram for concatenated decoder at $i$-th stage.

\section{Hard-Decision Multistage Decoding}

We now suggest a sub-optimal hard-decision multistage decoding algorithm [8, Section 15.2] for the case when all $\mathcal{R}_{i}$ are Gabidulin codes. Let $\mathcal{X} \subseteq\left(\mathbb{F}_{q}^{N \times T}\right)^{n}$ be a multishot matrix code obtained by the multilevel construction just described. Following the model in Section III, let $\left(\mathbf{X}_{0}, \ldots, \mathbf{X}_{n-1}\right) \in \mathcal{X}$ be the transmitted codeword and $\left(\mathbf{Y}_{0}, \ldots, \mathbf{Y}_{n-1}\right) \in\left(\mathbb{F}_{q}^{N \times T}\right)^{n}$ be the received sequence.

The multistage decoding occurs in $m$ stages; we start by finding the reduction $\left(\mathbf{r}_{j}, \mathbf{L}_{j}^{\prime}, \mathbf{E}_{j}^{\prime}\right) \in \mathbb{F}_{q^{M}}^{N} \times \mathbb{F}_{q}^{N \times \mu} \times \mathbb{F}_{q}^{\delta \times M}$ of each $\mathbf{Y}_{j} \in \mathbb{F}_{q}^{T \times M}$ and setting $\mathbf{r}_{j}^{(0)}=\mathbf{r}_{j}$. The decoding then proceeds in an iterative fashion. For $0 \leq i<m$, at the $i$-th stage, the following steps are executed (Figure 3 ).

1) Inner decoding. Using the generalized decoding method of Silva et al. [2], decode $\left(\mathbf{r}_{j}^{(i)}, \mathbf{L}_{j}^{\prime}, \mathbf{E}_{j}^{\prime}\right)$ into a codeword $\tilde{\mathbf{u}}_{j}^{(i)} \in \mathcal{R}_{i}$. Of course $\tilde{\mathbf{u}}_{j}^{(i)}$ belongs to some coset in $\mathcal{R}_{i} / \mathcal{R}_{i+1}$. Identify the leader $\tilde{\mathbf{v}}_{j}^{(i)} \in\left[\mathcal{R}_{i} / \mathcal{R}_{i+1}\right]$ of this coset and find-by inverse mapping - the message $\tilde{\mathbf{m}}_{j}^{(i)} \in \mathbb{F}_{q^{M}}^{K_{i}-K_{i+1}}$ that generated it.

2) After $n$ inner decodings we obtain $\left(\tilde{\mathbf{m}}_{0}^{(i)}, \ldots, \tilde{\mathbf{m}}_{n-1}^{(i)}\right)$. Similarly to the encoding procedure, define $\tilde{\mathbf{c}}^{(i)}$ as the vector in $\mathbb{F}_{p_{i}}^{n}$ corresponding to $\left(\tilde{\mathbf{m}}_{0}^{(i)}, \ldots, \tilde{\mathbf{m}}_{n-1}^{(i)}\right)$.

3) Outer decoding. Note that, due to errors, $\tilde{\mathbf{c}}^{(i)}$ may not be a codeword of $\mathcal{H}_{i}$. Therefore, decode $\tilde{\mathbf{c}}^{(i)}$ into the closest (in Hamming sense) codeword $\hat{\mathbf{c}}^{(i)} \in \mathcal{H}_{i}$.

At the end of the iteration, each $\mathbf{r}_{j}^{(i)}$ is updated to

$$
\mathbf{r}_{j}^{(i+1)}=\mathbf{r}_{j}^{(i)}-\hat{\mathbf{v}}_{j}^{(i)}
$$

where each $\hat{\mathbf{v}}_{j}^{(i)}$ is obtained from $\hat{\mathbf{c}}^{(i)}$ according to $\left[\mathcal{R}_{i} / \mathcal{R}_{i+1}\right]$ (just like in Steps 2 and 3 of the encoding procedure) and decoding proceeds to the next stage. After $m$ steps, we have $\hat{\mathbf{c}}^{(0)}, \ldots, \hat{\mathbf{c}}^{(m-1)}$. Figure 4 illustrates the whole process.

\section{CONCLUSION}

This work presented a bit more explicit multilevel construction of multishot codes for network coding than that introduced in [5]. A natural question arises: how good are the proposed codes? This demands a comparison of (9) with known bounds or previous one-shot constructions.

Another open problem is to adapt soft-decision multistage decoding algorithms [8, Section 15.3] to the current scenario. In particular, the metric is now the rank distance (as opposed to the Euclidean distance in the case of codes for the AWGN channel). This can possibly take advantage of list decoding of rank-metric codes.

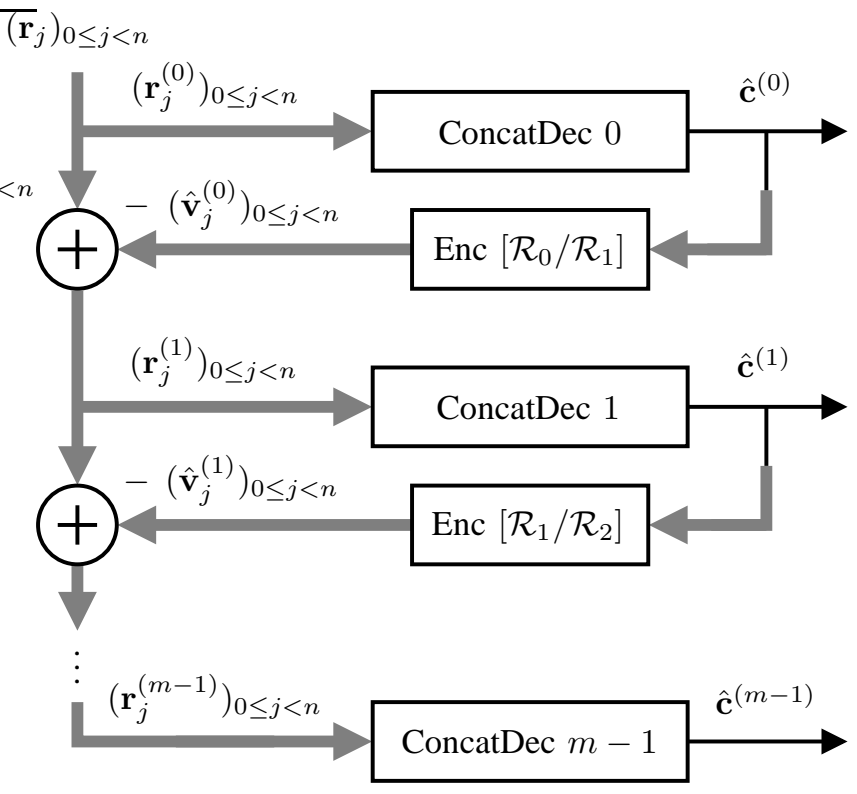

Figure 4. Block diagram for hard-decision multistage decoder. Each one of the ConcatDec blocks is detailed in Figure 3

\section{ACKNOWLEDGMENT}

The authors would like to thank Danilo Silva for helpful discussions, and CAPES (Brazil) and CNPq (Brazil) for bibliographical research and financial support.

\section{REFERENCES}

[1] R. Koetter and F. R. Kschischang, "Coding for errors and erasures in random network coding," IEEE Transactions on Information Theory, vol. 54, pp. 3579-3591, Aug. 2008.

[2] D. Silva, F. R. Kschischang, and R. Koetter, "A rank-metric approach to error control in random network coding," IEEE Transactions on Information Theory, vol. 54, pp. 3951-3967, Sept. 2008.

[3] E. M. Gabidulin, "Theory of codes with maximum rank distance," Problemy Peredachi Informatsii, vol. 21, no. 1, pp. 3-16, 1985.

[4] R. M. Roth, "Maximum-rank array codes and their application to crisscross error correction," IEEE Transactions on Information Theory, vol. 37, pp. 328-336, Mar. 1991.

[5] R. W. Nóbrega and B. F. Uchôa-Filho, "Multishot codes for network coding: Bounds and a multilevel construction," in Proceedings of the 2009 IEEE International Symposium on Information Theory (ISIT'09), (Seoul, South Korea), June 2009.

[6] H. Imai and S. Hirakawa, "A new multilevel coding method using errorcorrecting codes," IEEE Transactions on Information Theory, vol. 23, pp. 371-377, May 1977.

[7] E. L. Blokh and V. V. Zyablov, "Coding of generalized concatenated codes," Problemy Peredachi Informatsii, vol. 10, no. 3, pp. 45-50, 1974.

[8] S. Lin and D. J. Costello Jr., Error Control Coding. Pearson Prentice Hall, 2nd ed., 2004.

[9] D. Silva and F. R. Kschischang, "On metrics for error correction in network coding," IEEE Transactions on Information Theory, vol. 55, pp. 5479-5490, Dec. 2009. 